\title{
Polysomnography in a Large Population Based Study - the Study of Health in Pomerania Protocol
}

\author{
Beate Stubbe ${ }^{1 * \#}$, Thomas Penzel ${ }^{2 \#}$, Ingo Fietze ${ }^{2 \#}$, Anne Obst ${ }^{11}$, Carmen Garcia ${ }^{2}$, Sandra Zim- \\ mermann'2, Beate Diecker', Martin Glos ${ }^{2}$, Carsten Oliver Schmidt ${ }^{3}$, Katharina Lau ${ }^{3}$, Michael \\ Piontek $^{3}$, Katrin Hegenscheid ${ }^{4}$, Johannes Dober ${ }^{4}$, Klaus Berger ${ }^{5,6}$, Andras Szentkiralyi ${ }^{5}$, \\ Stephan B. Felix', Christoph Schäper', Sven Gläser ${ }^{1}$, Henry Völzke ${ }^{3,6,7}$ and Ralf Ewert ${ }^{1 "}$
}

${ }^{1}$ Department of Internal Medicine B - Cardiology, Pneumology, Infectious Diseases, Intensive Care Medicine, University Hospital Greifswald, Greifswald, Germany

${ }^{2}$ University Hospital Charité Berlin, Sleep Center, Berlin, Germany

${ }^{3}$ Department of Community Medicine, SHIP/Clinical Epidemiology Research, University Greifswald, Greifswald, Germany ${ }^{4}$ Institute of Clinical Radiology and Neuroradiology, University Hospital Greifswald, Greifswald, Germany

5Institute of Epidemiology and Social Medicine, University of Muenster, Muenster, Germany

${ }^{6}$ German Centre for Diabetes Research, DZD, Partner Site Greifswald, Germany

${ }^{7}$ German Centre for Cardiovascular Research, DZHK, Site Greifswald, Germany

\#These authors contributed equally to this work

*Corresponding author: Beate Stubbe (BS), MD, Department of Internal Medicine B, Cardiology, Pneumology, Infectious Diseases, Intensive Care Medicine, University Hospital Greifswald, F.-Sauerbruchstr, 17475 Greifswald, Germany, Tel: +49 383486 7242, Fax: +49 383486 7286, E-mail: beate.stubbe@uni-greifswald.de

\begin{abstract}
Background: Our current knowledge about sleep disorders in the general population is limited by a lack of objective sleep data assessed in large-scale, well characterised European cohorts.

The Study of Health in Pomerania (SHIP) is a population-based study investigating prevalence and incidence of common risk factors, subclinical disorders and clinical diseases in adults. Polysomnography (PSG) was performed between 2008 and 2012 (SHIP-TREND).

Aim: The aim of the present study was to perform cardiorespiratory PSG in a population- based setting to gain more insight into subclinical and clinical sleep disorders and their complex associations with common risk factors, subclinical disorders and chronic diseases among adults. Here we describe the rationale, design, and implementation of PSG in a population-based study.

Methods: The Study of Health in Pomerania (SHIP) consists of two independent population-based prospective subcohorts. The core diagnostic program of the baseline SHIP-TREND included the assessment of risk behaviour, common chronic diseases, cardiac, vascular, pulmonary, and serum blood parameters, mental health, and cognitive functioning. Genotyping and whole-body MRI were also performed. In addition, all participants were offered a standard overnight laboratory-based polysomnography (PSG). Subjective sleep parameters and restless legs syndrome (RLS) were assessed with a set of standardised questionnaires. Sleep staging, respiratory events, and leg movements were evaluated according to the standards of the American Academy of Sleep Medicine (AASM).
\end{abstract}

Results: Complete data (sleep-questionnaires, PSG) have been obtained on 1249 participants (573 women), aged 20-70 years. Genetic data are available from 424 PSG participants.

Conclusion: The population-based design and the comprehensive data collection provide a unique opportunity to assess the prevalence and risk factors of sleep disorders, and to investigate the complex relationships between sleep parameters, genetic factors, and various health and disease aspects.

\section{Keywords}

Epidemiology, Polysomnography, Genetics, Restless-legssyndrome

\section{Background}

Within the last 20 years technological progress of sleepmonitoring has improved diagnoses of sleep disorders [1]. The gold-standard for diagnosis and exclusion of sleep disorders is still the laboratory-based polysomnography (PSG). Besides improved technologies, standardised methods have greatly affected the quality of sleep recordings and were implemented into wideaccepted guidelines [1]. Sleep-related breathing disorders, such as obstructive sleep apnoea (OSA), are assumed to become further an increasing problem especially in the Western World as they are, besides diabetes, obesity and smoking, considered as relevant cardiovascular risk factors [2,3]. Large epidemiological studies have shown an association between OSAS and systemic hypertension in both general and sleep clinic populations [4-6]. However, many 
aspects of the relation between OSA and more specific cardiovascular risk factors are still unknown. Such factors are the extent of net blood pressure and other cardiopulmonary changes: the duration of OSA before inclusion, the percentage of hypertensive, overweight, and obese subjects, smoking status, alcohol use, and concomitant different medication therapy. In 1993, an estimated $2 \%$ of middleaged women and $4 \%$ of middle-aged men (30 to 60 years) met the minimal diagnostic criteria for obstructive sleep apnea syndrome (OSAS) [7]. These were the occurrence of more than 5 apnea and hypopnea events per hour of sleep (apnea hypopnea index, AHI) plus symptoms of excessive sleepiness. If the occurrence of apneas and hypopneas without symptoms are taken into account, then $9 \%$ of women and $24 \%$ of men suffered from an AHI above 5 events per hour of sleep. Currently the prevalence of OSA with or without self reported symptoms is estimated to be up to $5 \%$ of the population of the Western world [8]. However, a vast majority of affected individuals still remains undiagnosed $[9,10]$. In the planning phase of the current study, a limiting factor of sleep research was the lack of large, wellcharacterised population samples i.e., in Europe who underwent PSG. Meanwhile epidemiological studies performed in Switzerland [11] and the US [12] contribute to prevalence aspects of sleepdisorders, especially sleep disordered breathing. A recent study on sleep disordered breathing and heart failure contributes to still needed more population based studies investigating the relationship between cardiovascular risk indicators and the severity of sleep disordered breathing [13]. Longitudinal analyses on associations between sleep disorders, morbidity and mortality within epidemiological studies as well as a better understanding of the meaning of subclinical disorders are still needed.

Associations of genetics and diverse aspects of sleep disorders have been described previously $[14,15]$. However, thorough knowledge on possible associations is still of interest.

\section{Aims}

The main objectives of PSG measurements in SHIP-TREND were:

1. to estimate the prevalence of sleep disorders,

2. to assess subjective and objective sleep quality,

3. to assess longitudinal associations between sleep disorders, mortality, and morbidity and

4. to assess and better understand possible associations of sleep disordered breathing with age, gender, genetic factors, risk behaviour, and co-morbidities in a German adult populationbased cohort.

This paper outlines the study design and methods employed during the PSG assessments.

\section{Cohort Characteristics}

The Study of Health in Pomerania (SHIP) is a populationbased, large-scale cohort project with two main objectives: (i) to assess prevalence and incidence of common risk factors, subclinical disorders and clinical diseases, and (ii) to investigate their complex associations [16]. The study region for SHIP is West Pomerania in the northeast of Germany. The project consists of two independent cohorts: a two-stage stratified cluster sample of 6265 adults 20-79 years has been drawn in 1996 (SHIP-0), a five-year follow-up has been performed 2002-2006 (SHIP-1), a ten-year follow-up has been performed 2008-2012 (SHIP-2). A separate stratified sample of 10,000 subjects was drawn from the central population registry in the federal state of Mecklenburg-Vorpommern, Germany (SHIPTREND). Stratification variables were age, sex, and city/county of residence. Out of the net sample of 8826 , after exclusion of deceased and relocated subjects 4,420 (2,275 women) participated (response $50.1 \%)$. The invitation procedure included three written invitations, phone calls, and home visits $[16,17]$. Baseline data collection for SHIP-TREND started in 2008 and examinations were finalised in 2012. In comparison to SHIP-0, further examination methods were introduced in SHIP-TREND, including PSG. All SHIP-TREND participants were offered to take part in the PSG subproject. Before PSG examinations, the subjects have undergone baseline examinations (anthropometric measurements, blood sampling etc.) and have filled in a sleep questionnaire. The comprehensive interview and core examination program, including whole body magnetic resonance tomography (MRI), cardiorespiratory, sonographic, neurological, dermatological, dental and ophthalmological examinations were done before PSG. Blood and urine samples were obtained according to standardized procedures. Serum, EDTA and citrate plasma, DNA and urine are stored at $-80^{\circ} \mathrm{C}$ in a bio bank. In SHIP-TREND genotyping was performed in a subsample of 1000 subjects using Illumina Human Omni 2.5 Array (Illumina Inc., San Diego, CA, USA). For further details on the instruments of the SHIP and SHIPTREND examination program see Völzke et al. [16].

A SHIP-TREND follow-up, called SHIP-TREND-1, has started in March 2016. A follow-up on polysomnography within this framework is planned.

\section{PSG Examination}

All SHIP-TREND participants were offered to undertake a full night attended PSG. Before PSG examinations, the subjects underwent baseline examinations (anthropometric measurements, blood sampling etc.) and completed a set of questionnaires regarding their sleep.

Data collection of sleep-characteristics comprised of the following components:

1. baseline interview,

2. sleep questionnaires,

3. attended PSG.

Within the complex framework of the SHIP-TREND protocol, the night in the sleep laboratory complied specific regularities. Either the participants were directly transferred from the main examination centre to the out-of-hospital located sleep laboratory or an appointment was made as defined by the participant. The median time between the main investigation and the PSG performance was nine days. Due to acclimatisation purposes PSG started at the earliest two to three hours after arrival of the participants in the sleep laboratory.

\section{Interview}

Trained and certified interviewers conducted the computerassisted personal interviews, part of the baseline SHIP-TREND protocol (further details please see Völzke et al. [16]). Within the baseline interview five questions on sleep were included (Table 1).

\section{Standardized Sleep Questionnaires}

The standardized sleep questionnaires were handed to PSG participants only. The following scales were part of the sleep questionnaires (Table 2):

1. Epworth Sleepiness Scale (ESS) [18],

2. Insomnia Severity Index (ISI) [19-21],

3. Pittsburgh Sleep Quality Index (PSQI) [22],

4. Restless Legs Syndrome-Diagnostic Index (RLS-DI) [23-24].

Table 1: Sleep-questions of baseline SHIP-TREND interview.

\begin{tabular}{|c|l|}
\hline Question & \multicolumn{1}{|c|}{ Description } \\
\hline 1 & $\begin{array}{l}\text { Please assume the number of hours per night that you are asleep } \\
\text { on a regular day! }\end{array}$ \\
\hline 2 & Have you performed a midday nap within the last 7 days? \\
\hline 3 & $\begin{array}{l}\text { On how many times per week do you need more than } 30 \text { minutes } \\
\text { to fall asleep? }\end{array}$ \\
\hline 4 & $\begin{array}{l}\text { On how many times per week do you have problems to sleep } \\
\text { through? }\end{array}$ \\
\hline 5 & Do you snore? \\
\hline
\end{tabular}


Table 2: Standardized sleep questionnaires.

\begin{tabular}{|c|l|}
\hline Questionnaire & \multicolumn{1}{|c|}{ Description } \\
\hline $\begin{array}{c}\text { Epworth Sleepiness Scale } \\
\text { (ESS)13 }\end{array}$ & $\begin{array}{l}\text { The ESS is a scale intended to measure daytime sleepiness by use of a very short questionnaire. The questionnaire asks the } \\
\text { subject to rate his or her probability of falling asleep on a scale of increasing probability from 0 to } 3 \text { for eight different situations } \\
\text { [13]. The scores for the eight questions are added together to obtain a single number. A number in the 0-9 range is considered to } \\
\text { be normal while a number in the 10-24 range indicates that expert medical advice should be sought [13]. }\end{array}$ \\
\hline $\begin{array}{c}\text { Insomnia Severity Index 14-16 } \\
\text { The ISI is a seven-item self-report questionnaire. The seven answers are added up to get a total score. The scale assesses } \\
\text { sleep-onset and sleep maintenance difficulties, associated distress, and interference with daily functioning. Each item is rated } \\
\text { on a 0-4 scale. } \\
\text { The time period is the past fortnight. The guidelines for the interpretation of scores are: no clinically significant insomnia (0-7), } \\
\text { subthreshold insomnia (8-14), clinical insomnia of moderate severity (15-21) and severe clinical insomnia (22-28) [16]. }\end{array}$ \\
\hline $\begin{array}{c}\text { Pittsburgh Sleep Quality Index } \\
\text { (PSQI) [17,24] }\end{array}$ & $\begin{array}{l}\text { The Pittsburgh Sleep Quality Index (PSQI) is a self-rated questionnaire which assesses sleep quality and disturbances over a } \\
\text { 1-month time interval. Nineteen individual items generate seven "component" scores: subjective sleep quality, sleep latency, } \\
\text { sleep duration, habitual sleep efficiency, sleep disturbances, use of sleeping medication, and daytime dysfunction. The sum of } \\
\text { scores for these seven components yields one global score. A global PSQI score greater than 5 is supposed to distinguish good } \\
\text { and poor sleepers [17]. }\end{array}$ \\
\hline $\begin{array}{c}\text { Restless Legs Syndrome- } \\
\text { Diagnostic Index (RLS-DI)19 }\end{array}$ & $\begin{array}{l}\text { The RLS-DI consists of 10 items which are related to the essential diagnostic criteria established by the International RLS Study } \\
\text { Group (five items) as well as their supportive criteria (3 items) and features associated with RLS (2 items). Items have to be } \\
\text { completed using three categories per item that address frequency of occurrence of symptoms or certainty of presence or absence } \\
\text { of other diagnostic information. Within the present study only five items were explored. }\end{array}$ \\
\hline
\end{tabular}

Table 3: Variables of PSG.

\begin{tabular}{|l|}
\hline Data quality \\
\hline Light-on-time \\
\hline Light-off-time \\
\hline Total Sleep duration \\
\hline Time in bed \\
\hline Sleep efficiency \\
\hline Sleep latency \\
\hline Wake after sleep onset \\
\hline Sleep Stage N1\% total time asleep \\
\hline Sleep Stage N2\% total time asleep \\
\hline Sleep Stage N3\% total time asleep \\
\hline Sleep Stage REM \% total time asleep \\
\hline Arousals \\
\hline Arousal-Index \\
\hline Obstructive Apnoeas \\
\hline Mixed Apnoeas \\
\hline Central Apnoeas \\
\hline Hypopnoeas \\
\hline Apnoeas + Hypopnoeas \\
\hline Apnoea-Index \\
\hline Hypopnoea-Index \\
\hline Apnoea-Hypopnoea-Index \\
\hline Respiratory-Effort-Related-Arousals \\
\hline Respiratory-Effort-Related-Arousal-Index \\
\hline Oxygen-Desaturations $>4 \%$ \\
\hline
\end{tabular}

\section{Laboratory-Based Attended PSG}

Overnight attended PSG was conducted according to the standards of the American Academy of Sleep Medicine (AASM) [25] using ALICE 5 PSG devices (Philips Respironics, Eindhoven, The Netherlands). All sensors were carefully placed by trained and certified staff. Recordings included six channels of electroencephalogram (EEG, F4-M1, C4-M1, O2-M1, F3-M2, C3-M2, O1-M2, two channels of eletrooculogram (EOG), two channels of electromyogram (chin and tibialis muscles) [EMG], one electrocardiogram (ECG), inductive plethysmography detecting thoracic and abdominal movements, nasal pressure sensor, pulse oximetry, a microphone to detect snoring, and a body position sensor. Bio-calibration was performed before the light was switched-off (eye-movement, teeth grinding, breathing, snoring, and leg-movement). The variables documented or measured are listed in (Table 3). Up to four subjects were recorded in parallel per night. Participants were allowed to choose their own bedtime and to wake-up by themselves. However, due to reliability, a total in bed time of 8 hours was requested.

\section{PSG Analysis}

All PSG recordings were digitally stored and transferred to the University Hospital Charité, Center of Sleep Medicine Berlin, Germany, where sleep stages of 30-second epochs and respiratory events were evaluated visually by certified sleep technicians according to AASM 2007 criteria. Analysis of these data provided information on central, mixed, and obstructive sleep apnoeas, about hypopnoeas, periodic breathing, hypoventilation periods, sleep efficiency, sleep states, arousals, heart rate variability, posture during sleep, snoring, leg movements and events and degree of oxygen desaturations. The scoring of sleep stages was performed visually according to the AASM criteria. Sleep stages were determined in terms of minutes and percentages. The analysis of pathological breathing events including differentiation between central, mixed and obstructive apnoeas and differentiation between hypopnoeas, periodic breathing and hypoventilation periods was done visually according to established criteria from the AASM [25]. Respiratory related arousals were also visually scored. The apnoea-hypopnoea index was calculated as the number of apnoes/hypopnoeas per hour of sleep estimated by the total sleep time. The severity of sleep apnoea was defined as follows based on the Apnoea-Hypopnoea index (AHI):

No sleep apnoea - AHI $<5$ per hour sleep,

Mild sleep apnoea - AHI 5-15 per hour sleep,

Moderate sleep apnoea - AHI 15-30 per hour sleep,

Severe sleep apnoea - AHI $\geq 30$ per hour sleep.

Periodic leg movements and related arousals were detected using an automatic algorithm according to the AASM criteria [25]. The periodic leg movement index (PLMI) was calculated as the number of leg movements appearing periodically and meeting the amplitude criteria according to the PLM-definition per hour of sleep.

The pulse transit time (PTT) was calculated for each heart beat based on the finger plethysmographic signal derived from pulse oximetry and the ECG signal. The system for this study has already an algorithm implemented to calculate PTT in a continuous way. The heart rate variability was calculated from ECG derived beat-tobeat intervals. Standard heart rate variability parameters as the lowfrequency, the high-frequency component, and their ratio and mean heart rate values were calculated. In addition, new parameters derived using methods of statistical physics like long-range correlation, shortrange correlation, and entropy were calculated, too.

\section{MRI and PSG}

Data provided by whole body magnetic resonance imaging (MRI) were used to investigate the influence of anatomical structures on the genesis of disorders such as obstructive sleep apnea. Volunteers that took part in both examinations (PSG and MRI) were selected for measurements focusing on the retropalatal oropharynx region. Five cephalometric parameters were chosen for further investigation and comparison with PSG. Data on both PSG and MRI are available from 701 adults (341 women).

On the midsagittal plane: 
1. Minimal sagittal airway diameter- minimum distance between the posterior edge of the soft palate/uvula and the posterior pharyngeal wall.

On the transversal plane located at the mean distance between hard palate and tip of the uvula:

2. Lateral pharyngeal wall thickness- mean distance between the airway and the parapharyngeal fat pads.

3. Distance between the rami of the mandible-maximum distance between the inner sides of the most posterior parts of the rami of the mandible.

4. Distance between the fat pads-minimum distances between the parapharyngeal fat located lateral to the muscular pharyngeal wall.

5. Posterior airway wall thickness -distance between the airway and anterior edge of the spinal cord.

6. Intra- and inter-rater reliability was examined for chosen measurement methods and recorded data were archived in the SHIP database.

Further details on the MRI protocol are described by Hegenscheid et al. [26].

\section{Quality Assurance}

High-quality standards comprise an integral part of SHIP [1,27]. A standard operating procedure was established for PSG and MRI data measurement. The quality of sleep data was semi-annually discussed with independent scientists. Only certified personnel were allowed to conduct PSG. Initial certification of the PSG personnel was awarded to observers after a four weeks supervision by experienced personnel of the University Hospital Charité, Center of Sleep Medicine, Berlin, Germany. Furthermore, a 3-day education in the Center of Sleep Medicine Charité, Berlin was performed on the process of setting up and conducting PSG examination. During the study, certification procedures were repeated every 12 months, co-ordinated by the Sleep Centre Charité, Berlin. Inter- and IntraObserver variability was determined for the PSG data and for the MRI image measurements. Investigators were instructed by the radiology staff of the University Hospital Greifswald also in charge of the SHIP MRI. A quality monitoring system was applied to examinations and readings to detect and describe any kind of methodological irregularities including missing data, extreme values, observer, and device variability. Based on this system a quarterly feedback was provided, and if necessary actions were taken. Challenges relating to the inter-scorer reliability between different sleep centres have been described previously [28]. Due to the single-centre assessment of PSG data applied within SHIP the reliability of the data is considered as excellent. Two special aspects of the data quality control have to be highlighted:

1. During data collection, locations of PSG assessments had to be changed twice within the city of Greifswald. All aspects of relocation (dates, rooms, facilities) were documented. Methodical implications of these relocations on data quality were not detected.

2. Inter- and intra-scorer reliability control revealed conspicuities in the visual evaluation of sleep stages. Therefore it was necessary to re-evaluate a couple of hundreds PSG data sets. The re-evaluation was performed by a scorer with low intra-scorer variability.

The very complex quality control protocol ensures that the data, released for analysis purposes, are of excellent quality.

\section{Response and Attrition}

Considerable effort was made to maximize subject response for SHIP-Trend and the PSG participation [29]. Individuals who gave consent to participate in the PSG examination were approached by three invitational letters and repeated telephone calls. All PSG participants were provided a breakfast, a short medical report of the PSG results and free transport to the examination centre and back home.

From the 4420 subjects (2275 women) participating in SHIPTREND, 1630 individuals (754 women) volunteered for a PSG assessment (36.9\%). Finally, 1249 (573 women) spent the night in the sleep laboratory $(28.3 \%)$. Complete data on the parameters of breathing and EEG are available of 1209 individuals (559 women). Data on both PSG and MRI are available from 701 adults (341 women). Genetic data of the PSG participants are currently available from 424 individuals.

\section{Strengths and Weaknesses}

A major strength of the population-based SHIP-TREND is the comprehensiveness of information available on all aspects of sociodemography, health-status, risk factors and diseases in addition to polysomnographic sleep parameters. The SHIP-TREND is also notable for the high standards of quality assurance and the standardization of the applied methods. Much effort was put into personnel and structural resources. External facilities had to be rented only for the purpose of performing polysomnography within the framework of the study. Trained and certified students were deployed for the night watch. Separate work groups were responsible for data management and assessment.

A possible limitation is the potential selection bias due to the large proportion of individuals refusing to participate in the PSG assessment. In addition, the potential bias by selection to the PSG examination can be addressed by using the huge amount of data from the baseline examination. During data collection, locations of PSG investigations changed twice. However, all aspects of relocation (dates, rooms, facilities) were documented and methodical implications of these relocations on data quality were not detected. Finally, a single PSG night could be performed due to limited resources, whereas two consecutive nights would have been more optimal. We consider this a necessary trade-off in order to achieve a large number of PSG recordings in a population-based setting.

\section{Acknowledgements}

We gratefully acknowledge the contribution of field workers, study physicians, students and study nurses, computer scientists, medical documentarists and administration staff to data collection. Furthermore, we thank all study participants whose commitment and personal dedication have made this project possible.

\section{Statement of Interest}

The authors do not report any conflicts of interest.

\section{Author's Role}

All authors put substantial contribution to conception and design, analysis and interpretation of the data; on drafting the article and revising it critically for important intellectual content and final approval of the version to be published.

\section{Funding}

The work is part of the Community Medicine Research net (CMR) of the University of Greifswald, Germany, which is funded by the Federal Ministry of Education and Research (grants no. ZZ9603, 01ZZ0103, 01ZZ0403), Competence Network Asthma/ COPD (FKZ 01GI0881-0888), the Ministry of Cultural Affairs as well as the Social Ministry of the Federal State of Mecklenburg-West Pomerania. The CMR encompasses several research projects which are sharing data of the population-based Study of Health in Pomerania (SHIP; http:// ship.community-medicine.de). This work was additionally funded by the Deutsche RLS e.V. (German Restless Legs Syndrome Society).

\section{IRB Approval Statement}

The study conformed to the principles of the Declaration of Helsinki as reflected by the approval by the IRB / Ethics Committee 
of the University of Greifswald ("Leben und Gesundheit in Vorpommern", III UV 73/01).

\section{References}

1. (1999) Sleep-related breathing disorders in adults: recommendations for syndrome definition and measurement techniques in clinical research. The Report of an American Academy of Sleep Medicine Task Force. Sleep 22: 667-689.

2. Hiestand DM, Britz P, Goldman M, Phillips B (2006) Prevalence of symptoms and risk of sleep apnea in the US population: Results from the national sleep foundation sleep in America 2005 poll. Chest 130: 780-786.

3. Shahar E, Whitney CW, Redline S, Lee ET, Newman AB, et al. (2001) Sleepdisordered breathing and cardiovascular disease: cross-sectional results of the Sleep Heart Health Study. Am J Respir Crit Care Med 163: 19-25.

4. Nieto FJ, Young TB, Lind BK, Shahar E, Samet JM, et al. (2000) Association of sleep-disordered breathing, sleep apnea, and hypertension in a large community-based study. Sleep Heart Health Study. JAMA 283:1829-1836.

5. Lavie $P$, Herer $P$, Hoffstein $V(2000)$ Obstructive sleep apnoea syndrome as a risk factor for hypertension: population study. BMJ 320: 479-482.

6. Peppard PE, Young T, Palta M, Skatrud J (2000) Prospective study of the association between sleep-disordered breathing and hypertension. $\mathrm{N}$ Engl J Med 342: 1378-1384

7. Young T, Palta M, Dempsey J, Skatrud J, Weber S, et al. (1993) The occurrence of sleep-disordered breathing among middle-aged adults. N Engl J Med 328: 1230-1235.

8. Young T, Peppard PE, Gottlieb DJ (2002) Epidemiology of obstructive sleep apnea: a population health perspective. Am J Respir Crit Care Med 165 1217-1239.

9. Gibson GJ (2005) Obstructive sleep apnoea syndrome: underestimated and undertreated. Br Med Bull 72: 49-65.

10. Kapur V, Strohl KP, Redline S, Iber C, O'Connor G, et al. (2002) Underdiagnosis of sleep apnea syndrome in U.S. communities. Sleep Breath 6: 49-54.

11. Heinzer R, Vat S, Marques-Vidal P, Marti-Soler H, Andries D, et al. (2015) Prevalence of sleep-disordered breathing in the general population: the HypnoLaus study. Lancet Respir Med. 3: 310-318.

12. Alexander M, Ray MA, Hebert JR, Youngstedt SD, Zhang H, et al. (2016) The National Veteran Sleep Disorder Study: Descriptive Epidemiology and Secular Trends, 2000-2010. Sleep.

13. Javaheri S, Blackwell T, Ancoli-Israel S, Ensrud KE, Stone KL, et al. (2016) Sleep-disordered Breathing and Incident Heart Failure in Older Men. Am J Respir Crit Care Med 193: 561-568.

14. Gottlieb DJ, Hek K, et al. (2015) Novel loci associated with usual sleep duration: the CHARGE Consortium Genome-Wide Association Study. Mo Psychiatry 20: 1232-1239.
15. Szentkirályi A, Völzke H, Hoffmann W, et al. (2016) Lack of Association between Genetic Risk Loci for Restless Legs Syndrome and Multimorbidity. Sleep 39: 111-115.

16. Völzke H, Alte D, Schmidt CO, Radke D, Lorbeer R, et al. (2011) Cohort profile: the study of health in Pomerania. Int J Epidemiol 40: 294-307.

17. John U, Greiner B, Hensel E, Lüdemann J, Piek M, et al. (2001) Study of Health In Pomerania (SHIP): a health examination survey in an east German region: objectives and design. Soz Praventivmed 46: 186-194.

18. Johns MW (1991) A new method for measuring daytime sleepiness: the Epworth sleepiness scale. Sleep 14: 540-545.

19. Meisinger C, Heier M, Völzke H, Löwel H, Mitusch R, et al. (2006) Regional disparities of hypertension prevalence and management within Germany. J Hypertens 24: 293-299.

20. Bastien $\mathrm{CH}$, Vallières A, Morin CM (2001) Validation of the Insomnia Severity Index as an outcome measure for insomnia research. Sleep Med 2: 297-307.

21. Morin CM, Belleville G, Belanger L, Ivers H (2011) The Insomnia Severity Index: psychometric indicators to detect insomnia cases and evaluate treatment response. Sleep 34: 601-608.

22. Buysse DJ, Reynolds CF, Monk TH, Berman SR, Kupfer DJ (1989) The Pittsburgh Sleep Quality Index: a new instrument for psychiatric practice and research. Psychiatry Res 28: 193-213.

23. Berger K, von Eckardstein A, Trenkwalder C, Rothdach A, Junker R, et al. (2002) Iron metabolism and the risk of restless legs syndrome in an elderly general population--the MEMO-Study. J Neurol 249: 1195-1199.

24. Benes H, Kohnen R (2009) Validation of an algorithm for the diagnosis of Restless Legs Syndrome: The Restless Legs Syndrome-Diagnostic Index (RLS-DI). Sleep Med 10: 515-523.

25. Iber CAI, Chesson S A, Quan SF (2007) The AASM Manual for the Scoring of Sleep and Associated Events: Rules, Terminology and Technical Specifications. 1st ed. Westchester, Illinois American Academy of Sleep Medicine.

26. Hegenscheid K, Kuhn JP, Volzke H, Biffar R, Hosten N, et al. (2009) Wholebody magnetic resonance imaging of healthy volunteers: pilot study results from the population-based SHIP study. RoFo 181: 748-759.

27. Ludemann J, Piek M, Wood WG, Meyer S, Greiner B, et al. (2000) Methods for quality assurance of medical examination in epidemiological field studies: the "Study of Health in Pomerania" (SHIP). Gesundheitswesen 62: 234-243.

28. Penzel T, Zhang X, Fietze I (2013) Inter-scorer reliability between sleep centers can teach us what to improve in the scoring rules. J Clin Sleep Med 9: 89-91.

29. Haring R, Alte D, Völzke H, Sauer S, Wallaschofski H, et al. (2009) Extended recruitment efforts minimize attrition but not necessarily bias. J Clin Epidemiol 62: 252-260. 